\title{
An Efficient Way of Specifying Profile Inflow Boundary Conditions*
}

\author{
By Jianbo ZHANG ${ }^{1)}$ and Etsuo MORISHITA ${ }^{2)}$ \\ 1)Nissan Motors, Co. Ltd., Yokosuka, Japan \\ ${ }^{2}$ The University of Tokyo, Tokyo, Japan \\ (Received May 7th, 2003)
}

\begin{abstract}
An efficient way to systematically reproduce inflow profiles across a compressible, turbulent boundary layer for the mean flow variables, as well as the turbulent quantities in one-equation and two-equation turbulence-models, from given external flow conditions and one boundary layer parameter, is described. The reproduced profiles are checked against both experimental results and numerical solution of boundary layer equations. Theoretical analysis shows that a new form of density-weighted velocity, rather the Van Driest density-weighted velocity, obeys the linear-law at the viscous sublayer in a compressible turbulent boundary layer. Especially for non-adiabatic wall at hypersonic Mach numbers, where there are large density gradients, these two kinds of density-weighted velocity could differ considerably. Therefore, the new form of density-weighted velocity proposed in this paper should be employed for the viscous sublayer. It is also shown that power-law fitting for the streamwise velocity gives an unacceptable profile in the viscous sublayer. An efficient way to specify the normal velocity profile is also proposed and tested. The reproduced normal velocity at the boundary layer edge is found to agree remarkably well with the numerical solution of boundary layer equations.
\end{abstract}

Key Words: Boundary Conditions, Inflow Profiles, Compressible Flows, Computational Fluid Dynamics

\section{Nomenclature}

$a_{\mathrm{e}}$ : sound speed at boundary layer edge

$c_{\mathrm{f}}$ : local skin-friction coefficient $\left(=\tau_{\mathrm{w}} /\left(\rho_{\mathrm{e}} u_{\mathrm{e}}^{2} / 2\right)\right)$

$c_{\mathrm{p}}$ : specific heat at constant pressure

$H$ : shape factor or intermediate variable in Eq. (7)

$k$ : turbulent kinetic energy per unit mass

$L_{\text {ref }}$ : reference length scale

$M$ : Mach number

$n$ : exponent in the power-law for molecular viscosity

$N$ : exponent in the power-law fit for streamwise velocity

$P$ : pressure

$\operatorname{Pr}_{\mathrm{t}}$ : turbulent Prandtl number $(=0.9)$

$q$ : heat flux

$\left.R e\right|_{\mathrm{m}}$ : unit Reynolds number per meter

$R e_{\theta}$ : Reynolds number based on momentum thickness $\left(=\rho_{\mathrm{e}} u_{\mathrm{e}} \theta / \mu_{\mathrm{e}}\right)$

$R e_{\delta 2}$ : empirically-chosen Reynolds number, based on viscosity at the wall $\left(=\rho_{\mathrm{e}} u_{\mathrm{e}} \theta / \mu_{\mathrm{w}}\right)$

$T$ : temperature

$u$ : Farve-averaged streamwise velocity

$u^{+}=u / u_{\tau}$

$u_{\tau}:$ friction velocity $\left(=\left(\tau_{\mathrm{w}} / \rho_{\mathrm{w}}\right)^{1 / 2}\right)$

$u_{\mathrm{c}}$ : transformed velocity according to Eq. (2) or (10)

$v$ : Farve-averaged normal velocity

$x$ : streamwise coordinate

$y$ : distance above the wall

$y^{+}=y u_{\tau} / \nu_{\mathrm{w}}$

$\Delta x:$ streamwise integration step

$\varepsilon$ : dissipation rate

$\eta=y / \delta$ $\delta$ : boundary layer nominal thickness

$\delta^{*}$ : displacement thickness

$\Pi$ : profile parameter in Coles' formula

$\theta$ : momentum thickness

$\mu$ : molecular viscosity

$\mu_{\mathrm{t}}$ : turbulent eddy viscosity

$\nu$ : kinematic viscosity

$v^{\sim}$ : modeled quantity in Spalart-Allmaras's turbulence model

$\rho$ : mass density

$\tau$ : shear stress

$\tau_{x y}:$ principal turbulent shear stress

$\omega$ : specific dissipation rate

Subscripts

0 : stagnation condition

e: boundary layer edge

w: wall

\section{Introduction}

Specification of appropriate numerical boundary conditions around the solution domain is an important issue in the research of computational fluid dynamics (CFD). A typical numerical simulation usually involves four types of boundary conditions: inflow, outflow, farfield, and wall. For an object moving in the air, the inflow will be uniform. However, if one's interest is in a local area near the body, the issue of profile inflow boundary conditions will arise. When the size of the area of interest is comparable with the thickness of the upcoming boundary layer, the effect of inflow profile on the prediction result will become significant. While the treatment of boundary conditions at nearly uniform inflow, wall, farfield, and outflow ${ }^{1-6)}$ has reached a 
high degree of sophistication, the specification of solution profiles across a compressible, turbulent boundary layer at inflow is rather crude.

Currently, there are two main approaches to the specification of profile inflow conditions. Common practice simply fits the experimentally measured streamwise velocity with a power function, and calculates the temperature profile using Busemann-Crocco integral and the density profile through equation of state with the assumption of constant pressure across the boundary layer. This approach is straightforward and easy to program. However, power-law fits the velocity distribution well only in the outer region of a boundary layer. In the inner region, especially in the viscous sublayer, power-law is not a good fit. At the wall, it will give a singular velocity slope. One reason why people are not much disturbed by such crude treatment is the hope that the turbulent boundary layer will eventually develop to an equilibrium state by itself at some distance downstream. While this might be the case, it nonetheless requires extra length to be included in the inflow region of the solution domain. And it is not clear how long this development region should be.

A more sophisticated approach solves the compressible, turbulent boundary layer equations, coupled with a turbulence model, and uses the solution at the station that matches the experiment measurement as the inflow boundary conditions. This approach, while being quite accurate, is somewhat hard to implement. Developing a reliable turbulent boundary layer code itself might involve weeks of hard work.

What we proposed ${ }^{7)}$ is to use flow variable distributions reproduced from external conditions and one boundary layer parameter, through the use of the empirical law of wall, ${ }^{8}$ as the inflow conditions. This approach is much more accurate than the power-law fitting and much easier to implement than the solution of turbulent boundary layer equations. In this paper, three modifications to the procedures proposed in Ref. 8) will be outlined and tested against experimental results. Theoretical analysis will show that a new form of density-weighted velocity, rather than the Van Driest density-weighted velocity, obeys the linear-law in the viscous sublayer for high-speed flows. It will also be shown that power-law fitted stream-wise velocity distribution is unacceptable in the viscous sublayer.

It needs to be pointed out that the procedure proposed in Ref. 8) could reproduce the streamwise velocity distribution, but not the normal velocity distribution. And in common practice, normal velocity is usually assumed to be zero for convenience. However, it is demonstrated ${ }^{9)}$ the zero normal velocity assumption would lead to spurious expansion and compression waves near the inflow region. In this paper, an efficient way to reproduce normal velocity distribution inside boundary layer will be proposed.

For turbulence variables such as turbulence kinetic energy, turbulence dissipation rate or specific dissipation rate, empirical formulas simulating typical profiles in a boundary layer are employed in CFL3D. ${ }^{10)}$ In this paper, an efficient way to specify the turbulence inflow profiles that are consistent with the reproduced mean flow field will be proposed.

\section{Specification of Major Mean Flow Profiles}

A number of theories ${ }^{11-18)}$ has been developed to determine skin-friction and specify velocity profile for compressible turbulent boundary layers. The general consensus is that the Van Driest I transformation ${ }^{13}$ is a good fit to the experimental data of the velocity profile in the inner layer, and the Van Driest II transformation offers a good fit to the experimental data of skin friction. ${ }^{17)}$ Two limitations of Van Driest I transformation are that it is only valid for the log-law region and for a turbulence Prandtl number $\left(P r_{\mathrm{t}}\right)$ equal to one. The velocity profile of Huang et al. ${ }^{8)}$ is an extension of the Van Driest I transformation applied to Coles' profile ${ }^{12)}$ that includes the sublayer and the wake regions, and the transformation is modified to be valid for $P r_{\mathrm{t}}$ equal to 0.9 . This profile family is a good fit to the boundary layer velocity profiles for a wide range of Mach and Reynolds numbers. For completeness, the procedure proposed by Huang et al. will be briefly described first. Its modifications and extensions by the present authors are then followed.

\subsection{Brief description of Huang's procedures}

Theoretical analysis ${ }^{13)}$ and experimental evidence ${ }^{19)}$ suggest that the law-of-the-wall and the law-of-the-wake are transferable from incompressible flow to the compressible flow, provided that the velocity is defined by the densityweighted transformation and the wake parameter is correlated with empirically-chosen Reynolds number $R e_{\delta 2}$. The lawof-the-wall for the inner region, including the linear-law region, the buffer region and the log-law region, combined with Coles' law-of-the-wake for the outer region reads:

$$
\begin{aligned}
u_{\mathrm{c}}^{+} & =\frac{u_{\mathrm{c}}}{u_{\tau}}=u_{\mathrm{c}, \mathrm{b}}{ }^{+}+\frac{\Pi}{\kappa} w\left(\frac{y}{\delta}\right) \\
& =u_{\mathrm{c}, \mathrm{b}}{ }^{+}+\frac{\Pi}{\kappa}\left(1-\cos \left(\frac{\pi y}{\delta}\right)\right)
\end{aligned}
$$

where

$$
u_{\mathrm{c}}=\int\left(\frac{\rho}{\rho_{\mathrm{w}}}\right)^{1 / 2} \mathrm{~d} u
$$

and $u_{\mathrm{c}, \mathrm{b}}{ }^{+}$is a pure law-of-the-wall profile defined by

$$
\frac{\mathrm{d} u_{\mathrm{c}, \mathrm{b}}{ }^{+}}{\mathrm{d} y^{+}}=\frac{2}{\left(1+4 l^{+2}\right)^{1 / 2}+1}
$$

where

$$
l^{+}=\kappa y^{+}\left(1-e^{-y^{+} / A^{+}}\right), \quad \kappa \approx 0.41, \quad A^{+}=25.53
$$

The wake parameter $\Pi$ can be obtained from the curve fitting formula due to Cebeci and Smith ${ }^{20)}$ :

$$
\Pi=0.55\left[1-\exp \left(-0.24 \sqrt{R e_{\theta}}-0.298 R e_{\theta}\right)\right]
$$

As the pressure is nearly constant across the boundary layer, the density ratio in Eq. (2) can be replaced by a temperature ratio, which can be obtained by neglecting the con- 
vection terms and integrating the energy equation near a solid surface,

$$
T=T_{\mathrm{w}}-\frac{P r_{\mathrm{t}} q_{\mathrm{w}} u}{c_{\mathrm{p}} \tau_{\mathrm{w}}}-\frac{P r_{\mathrm{t}} u^{2}}{2 c_{\mathrm{p}}}
$$

The above equation establishes the relationship between $T_{\mathrm{w}}$ and $q_{\mathrm{w}} / \tau_{\mathrm{w}}$.

The integration of Eq. (2) yields the Van Driest transformation

$$
u_{\mathrm{c}}=\sqrt{B}\left[\sin ^{-1}\left(\frac{A+u}{D}\right)-\sin ^{-1}\left(\frac{A}{D}\right)\right]
$$

where

$$
\begin{aligned}
& A=q_{\mathrm{w}} / \tau_{\mathrm{w}} \\
& B=2 c_{\mathrm{p}} T_{\mathrm{w}} / P r_{\mathrm{t}} \\
& D=\sqrt{A^{2}+B}
\end{aligned}
$$

The inverse of Eq. (6) is

$$
\frac{u}{u_{\tau}}=\frac{1}{R} \sin \left(\frac{R u_{\mathrm{c}}}{u_{\tau}}\right)-H\left[1-\cos \left(\frac{R u_{\mathrm{c}}}{u_{\tau}}\right)\right]
$$

where

$$
\begin{aligned}
& R=u_{\tau} / \sqrt{B} \\
& H=A / u_{\tau}
\end{aligned}
$$

To obtain $c_{\mathrm{f}}$ and the corresponding boundary layer profiles for a given boundary layer displacement thickness or momentum thickness, the following iterative procedure needs to be performed:

1) Given $\delta^{*}$ (or $\theta$ ), guess $\delta^{*} / \delta, \theta / \delta$ and $u_{\tau}$ (or $\theta / \delta$ and $u_{\tau}$ ).

2) Calculate $\operatorname{Re}_{\delta 2}=\rho_{\mathrm{e}} u_{\mathrm{e}} \theta / \mu_{\mathrm{w}}$ and find $\Pi$ from Eq. (4).

3) Calculate $y_{\delta}{ }^{+}=u_{\tau} \delta / v_{\mathrm{w}}$ and obtain $u_{\mathrm{c}, \delta}{ }^{+}$from Eq. (1).

4) Obtain the nontransformed dimensionless velocity $u_{\delta}{ }^{+}$ from Eq. (7).

5) Update $u_{\tau}=u_{\mathrm{e}} / u_{\delta}{ }^{+}$and solve for $c_{\mathrm{f}}=2\left(T_{\mathrm{e}} / T_{\mathrm{w}}\right)$ $\times\left(u_{\tau} / u_{\mathrm{e}}\right)^{2}$.

6) Tabulate $u$ as a function of $\eta=y / \delta$ using Eqs. (1) and (7).

7) Update $\delta^{*} / \delta, \theta / \delta$ (or $\left.\theta / \delta^{*}\right)$ by performing the following integration numerically:

$$
\begin{aligned}
& \frac{\theta}{\delta}=\int_{0}^{1} \frac{\rho u}{\rho_{\mathrm{e}} u_{\mathrm{e}}}\left(1-\frac{u}{u_{\mathrm{e}}}\right) \mathrm{d} \eta \\
& \frac{\delta^{*}}{\delta}=\int_{0}^{1}\left(1-\frac{\rho u}{\rho_{\mathrm{e}} u_{\mathrm{e}}}\right) \mathrm{d} \eta
\end{aligned}
$$

where $\rho / \rho_{\mathrm{e}}$ is replaced by $T_{\mathrm{e}} / T$ with $T$ obtained from Eq. (5).

Steps 1 to 7 are repeated until the solution converges.

\subsection{Three modifications to Huang's procedures}

Three modifications to the above procedure are proposed to make it more convenient or more accurate.

\section{A. Use of an explicit expression for the wall velocity distribution due to Musker ${ }^{21)}$}

Equation (1) involves integration and is not convenient to use. In addition, there is a discrepancy in the slope condition at the edge of the boundary layer, as discussed by Cornish, ${ }^{22)}$ Bull, ${ }^{23)}$ and others. Instead, Musker's explicit expression for the law-of-the-wall at the inner region and law-of-the-wake at the outer region, satisfying the four boundary conditions: $y=0, u_{\mathrm{c}}=0$ and $\mathrm{d} u_{\mathrm{c}}{ }^{+} / \mathrm{d} y^{+}=1 ; y=\delta, u_{\mathrm{c}}=u_{\mathrm{ce}}$, and $\mathrm{d} u_{\mathrm{c}} / \mathrm{d} y=0$, can be used to facilitate the iterative procedure.

$$
\begin{aligned}
u_{\mathrm{c}}^{+}= & 5.424 \tan ^{-1}\left[\frac{2 y^{+}-8.15}{16.7}\right] \\
& +\log _{10}\left[\frac{\left(y^{+}+10.6\right)^{9.6}}{\left(y^{+^{2}}-8.15 y^{+}+86\right)^{2}}\right] \\
& -3.52+2.44\left\{\Pi\left[6\left(\frac{y}{\delta}\right)^{2}-4\left(\frac{y}{\delta}\right)^{3}\right]\right. \\
& \left.+\left(\frac{y}{\delta}\right)^{2}\left(1-\frac{y}{\delta}\right)\right\}
\end{aligned}
$$

\section{B. Velocity distribution at the viscous sublayer}

Velocity distribution at the viscous sublayer for compressible turbulent boundary layer needs further examination. In Coles' original proposal for Eq. (1), the viscous sublayer is neglected. Huang et al. ${ }^{8}$ ) found that it is important to include the sublayer contribution for high-speed flows because the sublayer becomes thicker and may occupy a substantial portion of the whole boundary layer at hypersonic Mach numbers. In using Eq. (1) as the base for their profile family, it is actually assumed that Van Driest transformed velocity, Eq. (2), obeys the same linear-law as that in the incompressible case. They acknowledge that they cannot claim detailed reliability of their profile family in the sublayer. As sublayer data for compressible flows are scarce, especially for large heat transfer rates, theoretical analysis will be pursued to examine this problem in the following.

As in the incompressible turbulent boundary layer, there is a thin layer of constant total shear stress, called the inner layer, in a compressible turbulent boundary layer. In the turbulent core region, the laminar shear stress is dominated by the Reynolds stress and hence can be neglected. By assuming $\operatorname{Pr}_{\mathrm{t}}$ equal to one and invoking Prandtl's mixing length theory, Van Driest ${ }^{13)}$ showed that the transformed velocity obeys the same log-law as that in the incompressible case. In the immediate vicinity of the wall, due to the damping of the wall, Reynolds stress can be neglected. The laminar shear stress equals the shear stress at the wall:

$$
\mu \frac{\partial u}{\partial y}=\tau_{\mathrm{w}}
$$

Divided by viscosity at the wall, it becomes:

$$
\frac{\mu}{\mu_{\mathrm{w}}} \frac{\partial u}{\partial y}=\frac{\rho_{\mathrm{w}} u_{\tau}^{2}}{\mu_{\mathrm{w}}}
$$

Integrating and using power-law for molecular viscosity:

$$
\frac{\int\left(\frac{T}{T_{\mathrm{w}}}\right)^{n} \mathrm{~d} u}{u_{\tau}}=y^{+}
$$


Table 1. Comparison of reproduced skin friction coefficient, momentum and nominal thickness, and shape factor against the experimental values (The figures in the parenthesis are the errors relative to the experiment).

\begin{tabular}{lccc}
\hline & $c_{\mathrm{f}}$ & $\theta(\mathrm{cm})$ & $\delta(\mathrm{cm})$ \\
\hline Given $\theta$ & $8.0 \mathrm{E}-04(5.2 \%)$ & & $1.53(13.3 \%)$ \\
Given $\delta$ & $8.4 \mathrm{E}-04(10.5 \%)$ & $0.0357(-23.0 \%)$ & $18.0(-4.2 \%)$ \\
Experiment $^{18)}$ & $7.6 \mathrm{E}-04$ & 0.0464 & 1.35 \\
\hline
\end{tabular}

As before, the temperature ratio in Eq. (9) can be replaced by the density ratio:

$$
\frac{\int\left(\frac{\rho}{\rho_{\mathrm{w}}}\right)^{-n} \mathrm{~d} u}{u_{\tau}}=y^{+}
$$

It indicates that the velocity transformed according to Eq. (9) or (10) rather than Eq. (2) obeys the linear-law for high-speed flows. For an adiabatic wall, the temperature, hence the density, is nearly constant in the viscous sublayer, therefore, these two kinds of transformation give essentially the same results. However, for a non-adiabatic wall, especially for large heat transfer at hypersonic Mach numbers, large density gradient exists at the sublayer. These two kinds of transformation can be quite different. Therefore, for a non-adiabatic wall, Eq. (9) or (10) should be invoked to modify the streamwise velocity after obtaining $u_{\tau}$ through the iterative procedure described above. For a more accurate streamwise velocity distribution, Sutherland's law, which is a better fit than power-law for molecular viscosity, can be used instead of the power-law in Eq. (9).

The actual calculation consists of the following three steps.

1) Determine $u_{\tau}$ through the iterative procedure.

2) Substitute Crocco's integral, Eq. (5), into Eq. (9), integrate it numerically, thereby establishing $y$ as a tabulated function of $u$.

3) Interpolate this tabulated function to find $u$ at the desired value of $y$.

C. Reproducing velocity distribution from boundary layer thickness $\delta$

In the situation where the boundary layer thickness $\delta$ is known or provided, rather than $\delta^{*}$ or $\theta$, the following five steps could replace the first five steps in Huang's algorithm:

1) Given $\delta$, guess $\theta$ and $u_{\tau}$, and calculate $\tau_{\mathrm{w}}$.

2) Calculate $u_{\text {ce }}$ from Eq. (6), calculate $R e_{\delta 2}=\rho_{\mathrm{e}} u_{\mathrm{e}} \theta / \mu_{\mathrm{w}}$ and find $\Pi$ from Eq. (4).

3) Calculate $\operatorname{Re}_{\delta, \mathrm{w}}=\rho_{\mathrm{w}} u_{\mathrm{ce}} \delta / \mu_{\mathrm{w}}$.

4) Solve for $y_{\delta}{ }^{+}$from $\operatorname{Re}_{\delta, \mathrm{w}}=\left(u_{\mathrm{ce}} / u_{\tau}\right)\left(\rho_{\mathrm{w}} u_{\tau} \delta / \mu_{\mathrm{w}}\right)=$ $u_{c \delta}{ }^{+} y_{\delta}{ }^{+}$and Eq. (8).

5) Update $u_{\tau}=y_{\delta}{ }^{+} \mu_{\mathrm{w}} /\left(\rho_{\mathrm{w}} \delta\right)$, and solve for $c_{\mathrm{f}}=$ $2\left(T_{\mathrm{e}} / T_{\mathrm{w}}\right)\left(u_{\tau} / u_{\mathrm{e}}\right)^{2}$.

\subsection{Test of the effectiveness of the modifications}

To test the effectiveness of these modifications in reproducing the compressible turbulent boundary layer profiles, the experimental results in Ref. 18) are chosen as a benchmark. The external flow conditions are as follows:

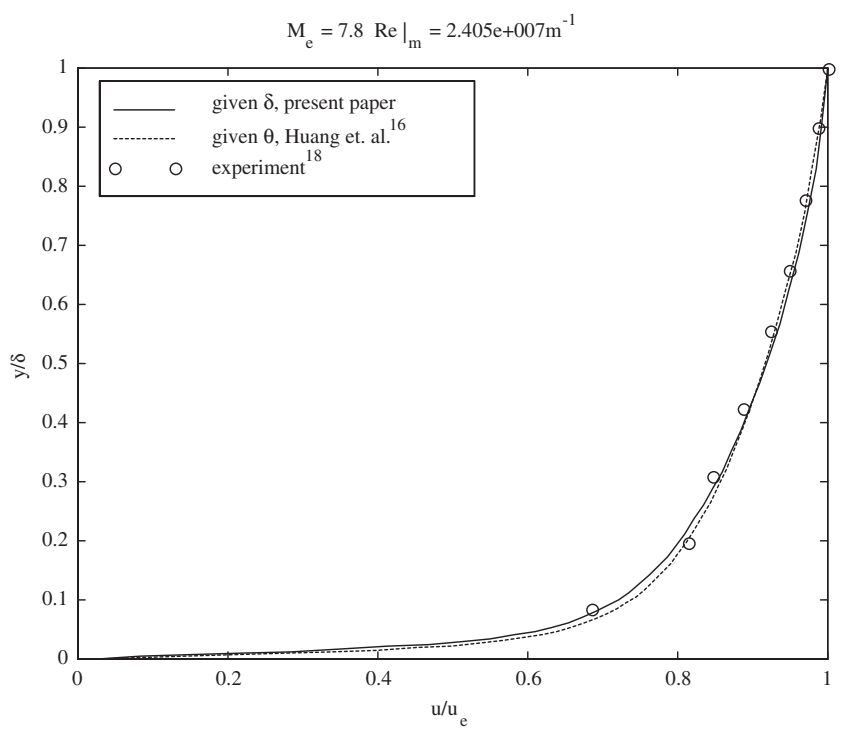

Fig. 1. Comparison of reproduced streamwise velocity profiles with experiment values.

$$
\begin{aligned}
& M_{\mathrm{e}}=7.80,\left.\quad R e\right|_{\mathrm{m}}=24.05 \times 10^{6} \mathrm{~m}^{-1}, \\
& P_{\mathrm{e}}=1230 \mathrm{~Pa}, \quad T_{0}=688 \mathrm{~K}, \quad T_{\mathrm{w}}=306 \mathrm{~K}
\end{aligned}
$$

Figure 1 compares the streamwise velocity profiles reproduced from a given $\theta$ or $\delta$ against that measured from experiment. The reproduced $c_{\mathrm{f}}, \delta$ or $\theta$ are compared with the experimental results in Table 1 . It is clear that better agreement with experimental results is obtained when $\theta$ is given. This could be attributed to the fact the boundary layer momentum thickness $\theta$ is an integral parameter and hence could be determined with higher accuracy through experiment, while boundary layer thickness is not an integral parameter and can only be estimated with high uncertainty. Another difference between $\theta$ and $\delta$ is that boundary layer momentum thickness contains information on both velocity and density, while boundary layer thickness contains only information on velocity.

Figure 2 shows the reproduced density and temperature profiles across the boundary layer. It has to be noted that any usage of Crocco's integral, even with the inclusion of a recovery factor to take into account the realistic turbulent Prandtl number, implies that velocity boundary layer thickness is equal to temperature boundary layer thickness, which is not true for compressible flows, especially for hypersonic flows.

Figure 3 compares the streamwise velocity distribution, in a wall unit, in the viscous sublayer. The solid line depicts the streamwise velocity distribution according to Eq. (10), 


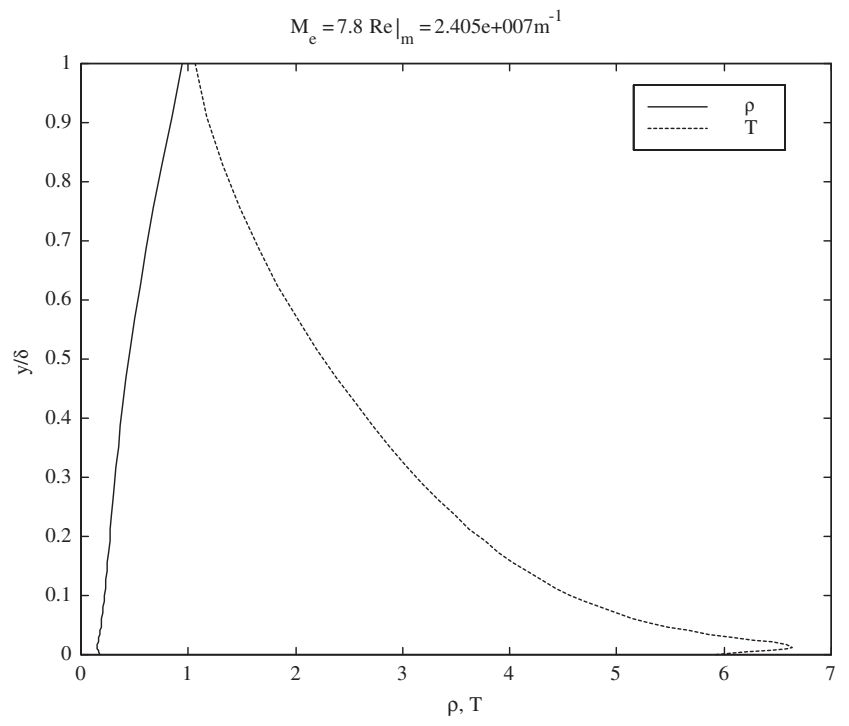

Fig. 2. Reproduced density and temperature profiles (Given $\theta$ ).

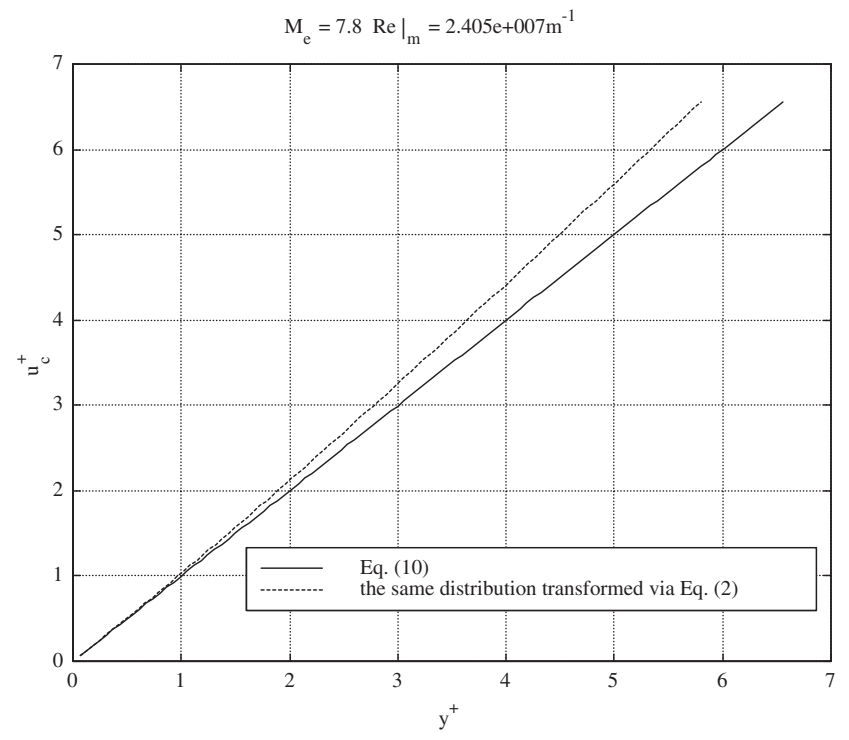

Fig. 3. Reproduced velocity distribution in viscous sublayer for hypersonic flow with iso-thermal wall.

while the dashed curve depicts the same distribution but transformed according to Eq. (2). It is clear that a difference of about $10 \%$ at the outer edge of viscous sublayer $\left(y^{+}=5\right)$ exists between the two transformations. Although the distribution according to Van Driest transformation still closely resembles a straight line, its slope is quite different from linear-law in the incompressible case. Since reliable data on streamwise velocity at viscous sublayer in a hypersonic boundary layer is not readily available, no attempt at present is made to compare the theoretical result of this paper, Eq. (10), with any experimental result.

\subsection{Observation on the use of power-law velocity pro- file in the viscous sublayer}

As noted in the introduction, it is common practice in an experiment to fit velocity distribution across the boundary layer to power functions. In CFD, power-law velocity distri-

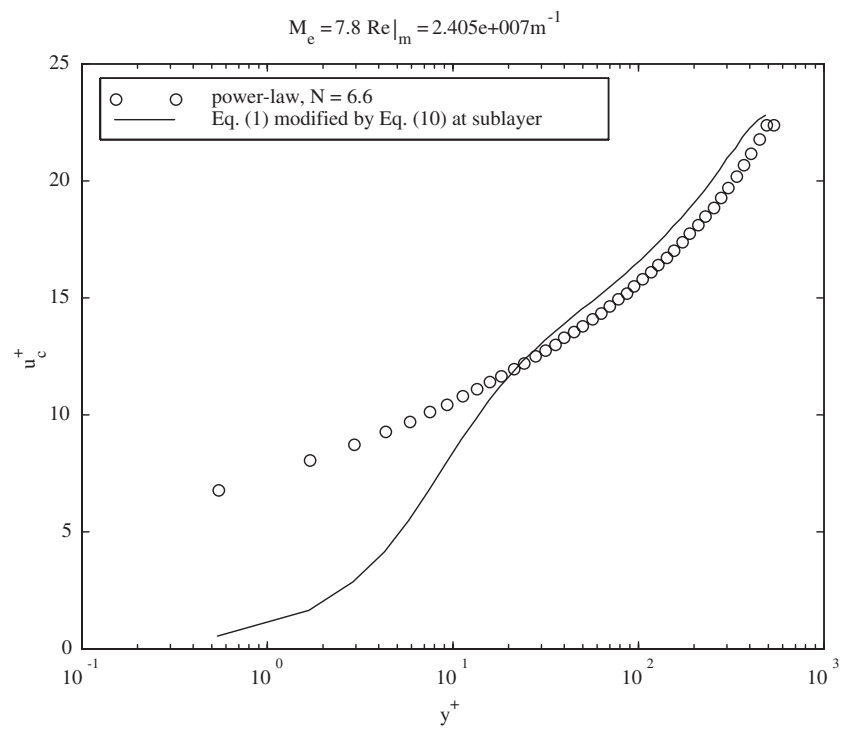

Fig. 4. Comparison of reproduced velocity profiles in wall unit between power-law and law-of-the-wall (Power-law unacceptable in inner layer).

bution is often used as a convenience to set the inflow streamwise velocity profile. However, power-law is only approximately applicable to the outer part of the boundary layer. In the immediate vicinity of the wall, power-law assumption will result in qualitatively incorrect velocity distribution. In fact, at the wall, the normal derivative of streamwise velocity according to the power-law is singular. Two-equation turbulence models usually require the $y^{+}$at the first grid point be less than 1 , and there should be several grid points within the viscous sublayer, if wall-function approach is not used. In such a situation, the streamwise velocity distribution near the wall should be determined through the iterative procedure described above, rather than using power-law fitting. Figure 4 compares the velocity distributions in sublayer, in wall units, obtained from experimentally fitted powerlaw and that reproduced from the law-of-the-wall as analyzed in the subsection B. It is clear that the distribution obtained from the power-law is totally unacceptable in the viscous sublayer.

\section{Specification of Normal Velocity Profile}

Normal velocity within the boundary layer is deemed sufficiently small and usually assumed to be zero for convenience. However, Orkwis et al. ${ }^{9)}$ demonstrate in the simulation of consecutive flat plates that spurious shock and expansion waves will be generated as a result of the zero normal velocity assumption in the inflow profile for the second plate. To the authors' limited knowledge, there has been no efficient way of specifying a normal velocity profile for a compressible turbulent boundary layer in the open literature. In this paper, by incorporating the continuity equation and integral momentum equation with the iterative procedure in section 2, a way to determine the normal velocity profile is proposed and tested against the boundary layer solution.

The Favre-averaged ${ }^{24)}$ continuity equation for steady 
compressible flow reads

$$
\frac{\partial \rho u}{\partial x}+\frac{\partial \rho v}{\partial y}=0
$$

It follows that if the density and streamwise velocity profile at two closely located stations are known, the normal velocity profile can be obtained through the integration of Eq. (11). It is already shown in section 2 that the density and streamwise velocity profile at a station can be efficiently reproduced, with good accuracy, as long as the external flow conditions and one boundary layer parameter at that station are provided. The question left is how to find the density and streamwise velocity profile at the next station. For this purpose, a momentum integral equation for a boundary layer over a flat plate under zero-pressure gradient can be employed.

$$
\frac{\mathrm{d} \theta}{\mathrm{d} x}=\frac{c_{\mathrm{f}}}{2}
$$

The procedure to specify normal velocity profile consists of the following steps:

1) Given the external flow conditions and one integral parameter, say, boundary layer momentum thickness $\theta$ at one station, calculate the density, streamwise velocity profile, and skin friction coefficient $c_{\mathrm{f}}$ at that station through the algorithm described in section 2 .

2) Determine the boundary layer momentum thickness at the next station through the integration of Eq. (12).

3) Calculate the density, streamwise velocity profile at the new station in the same manner as in step 1.

4) Determine the normal velocity profile through the integration of Eq. (11).

To check the effectiveness of the above procedure, the sonic flow over an isothermal flat plate, employed by Wil$\operatorname{cox}^{25)}$ to teach the usage of his boundary layer solution program EDDYBL, is chosen as the benchmark. The external flow conditions are as follows:

$$
\begin{aligned}
& M_{\mathrm{e}}=1.0,\left.\quad R e\right|_{\mathrm{m}}=4.07 \times 10^{6} \mathrm{~m}^{-1}, \\
& P_{0}=23112 \mathrm{~Pa}, \quad T_{0}=260 \mathrm{~K}
\end{aligned}
$$

The temperature at wall is $242 \mathrm{~K}$. The station chosen for comparison has $c_{\mathrm{f}}=2.704 \times 10^{-3}$, and $\theta=1.527 \times$ $10^{-3} \mathrm{~m}$.

The normal velocity profile is not an output in Wilcox's program. However, the normal velocity at the boundary layer edge $\left(v_{\mathrm{e}}\right)$ can be obtained from the integration of the continuity equation using the solution of boundary layer equations at two adjacent stations. The value is found to be $0.6395 \mathrm{~m} / \mathrm{s}$, and can be taken as a close approximate to the exact value. The corresponding value reproduced from the proposed procedure is $0.673 \mathrm{~m} / \mathrm{s}$. It is remarkable that the difference is only about $5 \%$ of the exact value, considering the fact that $v_{\mathrm{e}}$ is only about $0.2 \%$ of the freestream velocity. The reproduced normal velocity profile is shown in Fig. 5. The comparison of the reproduced profiles for density, streamwise velocity, and temperature with the boundary layer solutions, is shown in Fig. 6. Comparison of skin fric-

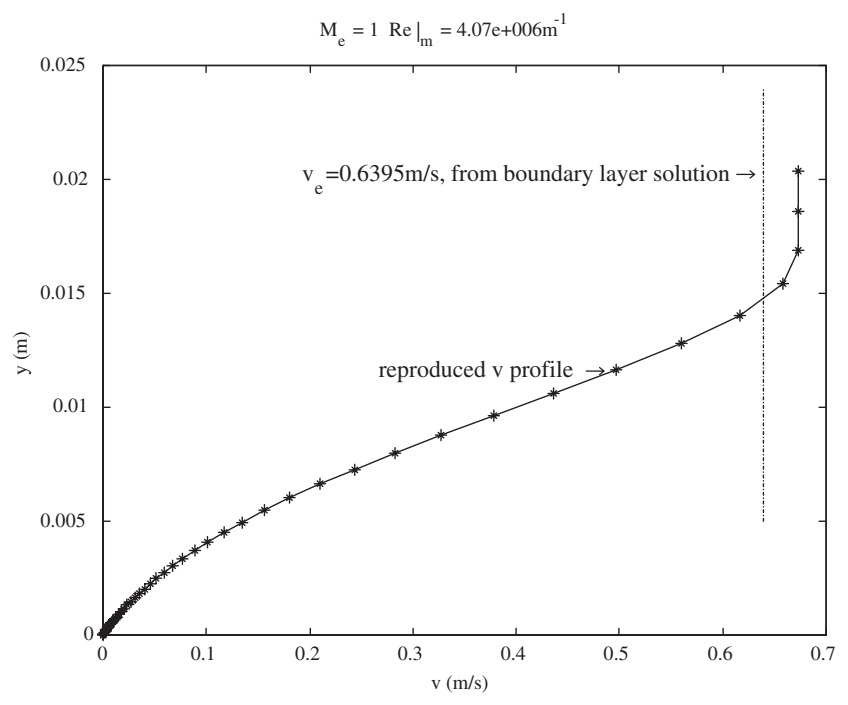

Fig. 5. Reproduced normal velocity profile.

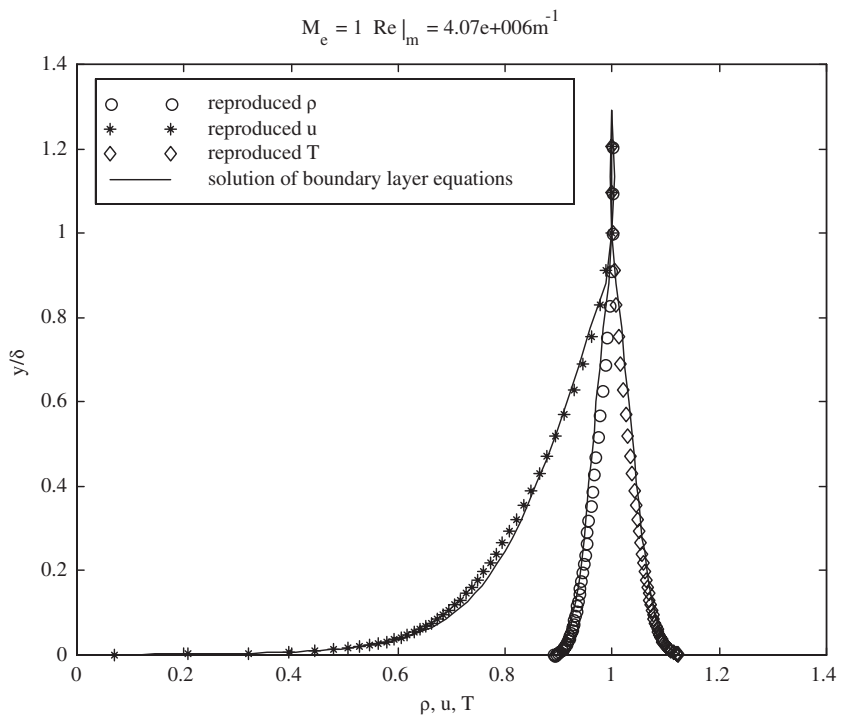

Fig. 6. Comparison of reproduced profiles and solution of boundary layer equations.

Table 2. Comparison of reproduced skin friction coefficient, shape factor and normal velocity at the edge of boundary layer against the solution of $\mathrm{BL}$ equations. (The figures in the parenthesis are the errors relative to the BL solution.)

\begin{tabular}{lccc}
\hline & $c_{\mathrm{f}}$ & $H$ & $v_{\mathrm{e}}(\mathrm{m} / \mathrm{s})$ \\
\hline Reproduced & $2.66 \mathrm{E}-03(-1.5 \%)$ & $1.68(-1.2 \%)$ & $0.6730(5.2 \%)$ \\
Solution of BL eqs & $2.70 \mathrm{E}-03$ & 1.70 & 0.6395 \\
\hline
\end{tabular}

tion coefficient, shape factor, and normal velocity at boundary layer edge is listed in Table 2 .

A word of caution for the choice of streamwise step $\Delta x$ in the integration of Eq. (12) is in order. It should neither be too large, to incur excessive discretization error, nor be too small, to incur round-off error. In the example above, non-dimensionalized $\Delta x$ from 0.01 to 0.5 gave essentially the same results. 


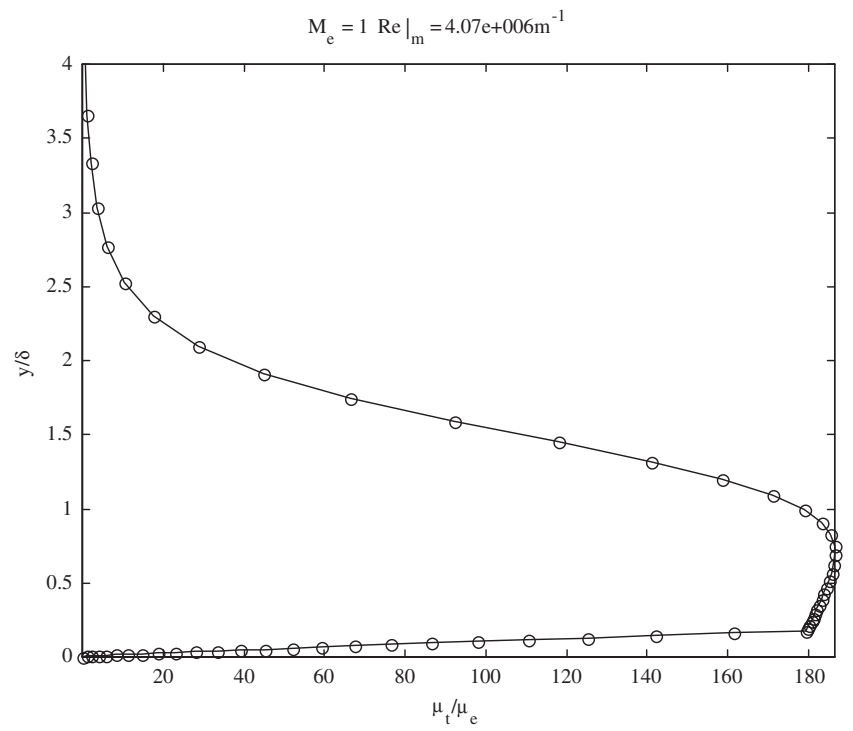

Fig. 7. Profiles of turbulent viscosity reproduced from Baldwin-Lomax algebraic turbulence models.

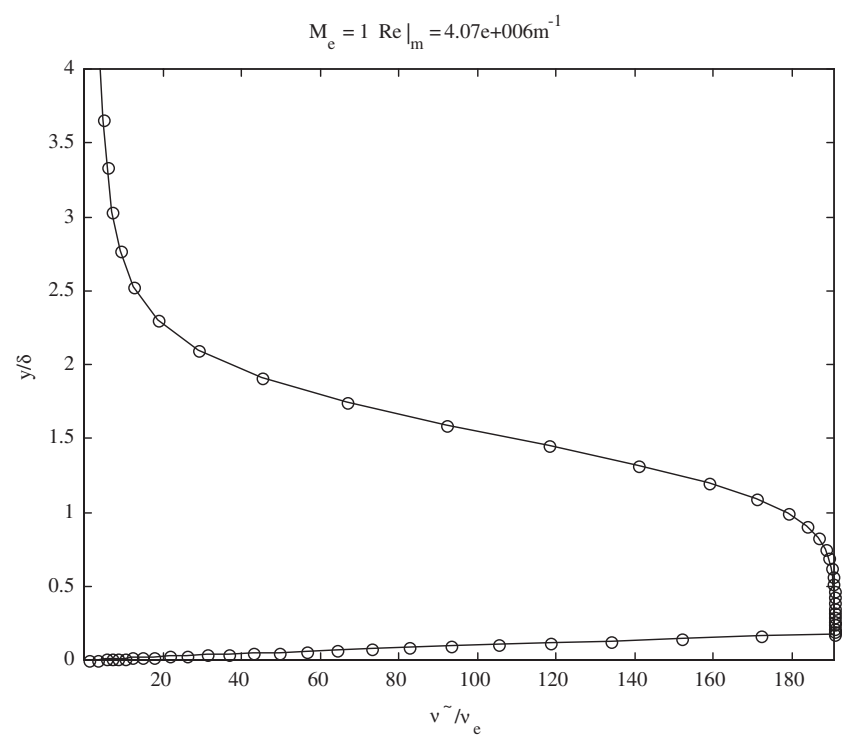

Fig. 8. Reproduced profile of the modeled quantity in Spalart-Allmaras's one-equation turbulence model.

\section{Specification of Turbulence Quantity Profiles}

With the mean flow profiles of density, velocity, and temperature available, turbulent quantity profiles can be determined either from algebraic turbulence models, from experimentally established laws, or from their definitions in turbulence models.

1) The turbulent viscosity profile can be readily calculated with any simple algebraic turbulence model, say Baldwin-Lomax's model. ${ }^{26)}$

2) For the one-equation turbulence model of Spalart-Allmaras ${ }^{27)}$ the modeled quantity is related to the turbulent viscosity via an algebraic equation, and hence can be determined from the solution of that equation.

3 ) For two-equation turbulence models, ${ }^{28-30)}$ first the principal turbulence shear stress $\left(\tau_{x y}\right)$ is calculated as

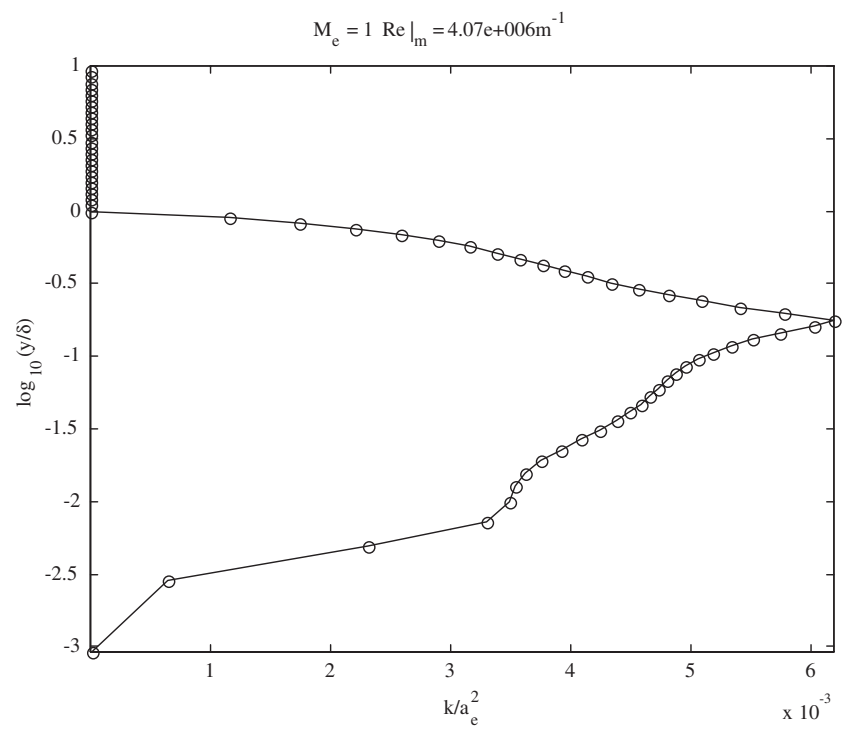

Fig. 9. Reproduced profile of turbulent kinetic energy.

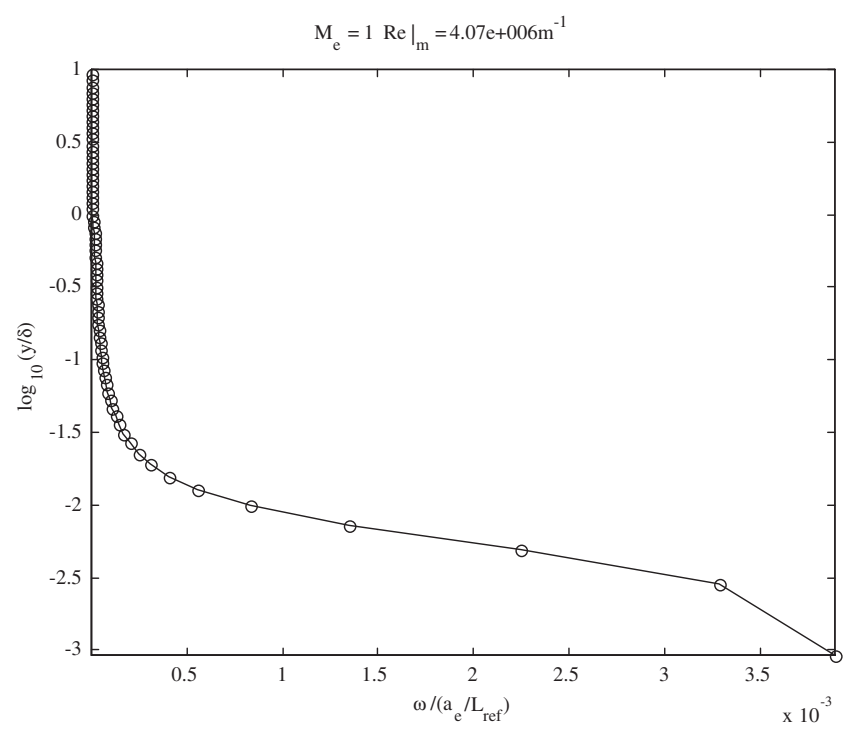

Fig. 10. Reproduced profile of turbulent specific dissipation rate.

the product of the turbulent viscosity and the strain rate of the mean flow (neglecting the velocity derivative along the flow direction). Then, turbulent kinetic energy $k$ can be determined through the experimentally established relation $^{31)} \tau_{x y}=a k$, where $a$, the so-called structural parameter, has a value of about 0.3 .

4) The turbulence dissipation rate $(\varepsilon)$ or specific dissipation rate $(\omega)$ can be deduced from $k$ and turbulent viscosity according to their relationship in the turbulence models.

5) Within the viscous sublayer, there is no proportionality between $\left(\tau_{x y}\right)$ and $k$. Instead, asymptotic results from Taylor series expansion and Navier-Stokes equations applied in the vicinity of wall can be employed. The leading term of turbulent kinetic energy varies quadratically with the normal distance from the wall, the coefficient of which can be determined by matching the so- 
lutions in the near wall region with that in the region away from the wall. The analytical formula for specific dissipation rate $(\omega)$ in the vicinity of wall exhibits an inversely quadratic behavior.

The profiles of turbulent viscosity $\left(\mu_{\mathrm{t}}\right)$, the quantity modeled in Spalart-Allmaras's one-equation turbulence model, turbulent kinetic energy $(k)$, and specific turbulent dissipation rate $(\omega)$, corresponding to the mean flow profiles in Fig. 6, are shown in Figs. 7-10, respectively.

\section{Conclusions}

The problem of specifying profile inflow boundary conditions, which occurs when the dimension of the solution domain is comparable with the thickness of the upcoming boundary layer, is an area that has not received adequate attention. Instead of using power-law fitting or a solution of coupled boundary layer equations, we proposed to use the reproduced profiles from external conditions and one boundary layer parameter as the profile inflow boundary conditions. In this paper, three modifications are proposed to make the iterative procedure devised in Ref. 8) either more convenient or more accurate.

First, in place of the extended Coles's profile, which requires integration, it is proposed to use Musker' ${ }^{21)}$ explicit expression for the wall velocity distribution to facilitate the iterative procedure.

Second, theoretical analysis shows that a new form of density-weighted velocity, rather the Van Driest densityweighted velocity, obeys the linear-law at the viscous sublayer in a compressible turbulent boundary layer. Especially for nonadiabatic wall at hypersonic Mach numbers, where there are large density gradients, these two kinds of density-weighted velocity could differ considerably. The linearlaw for the new kind of density-weighted velocity should be employed to modify the profiles at the sublayer.

Third, an iterative procedure is proposed for the case when boundary layer nominal thickness, rather than the displacement or momentum thickness, is given. This is useful in the situation where only the value of boundary layer nominal thickness is available.

The effects of these modifications are checked against experimental results of a turbulent, hypersonic boundary layer. It is found that boundary layer momentum thickness leads to a better agreement with experimental results than boundary layer thickness. Therefore, boundary layer momentum thickness, when available, should be preferred in the reproducing procedure. It is also shown that power-law fitting for the streamwise velocity gives unacceptable profile in the viscous sublayer.

Normal velocity inflow profile could have significant effect on the simulation results. For the first time, to the best knowledge of the authors, a way to reproduce normal velocity profile is proposed by incorporating the continuity equation and boundary layer integral momentum equation with the above iterative procedure. The reproduced normal velocity at the boundary layer edge is found to agree remark- ably well with the numerical solution of the boundary layer equations.

Besides the mean flow variable profiles, ways to specify profiles for turbulent quantities that are consistent with mean flow, based on established experimental observations, are also proposed.

The same idea in this paper can readily be used in the determination of inflow profiles involving an incompressible boundary layer.

\section{References}

1) Thomas, J. L. and Salas, M. D.: Far Field Boundary Conditions for Transonic Lifting Solutions to the Euler Equations, AIAA J., 24 (1986), pp. 1074-1080.

2) Giles, M. B.: Nonreflecting Boundary Conditions for Euler Equation Calculations, AIAA J., 28 (1990), pp. 2050-2058.

3) Pulliam, T. H. and Steger, J. L.: Recent Improvements in Efficiency, Accuracy and Convergence for Implicit Approximate Factorization Algorithms, AIAA 23rd Aerospace Sciences Meeting, AIAA Paper 85-0360, 1985.

4) Nordstrom, J.: Extrapolation Procedures for the Time-Dependent Navier-Stokes Equations, AIAA J., 30 (1992), pp. 1654-1656.

5) Rizzi, A. W.: Numerical Implementation of Solid-Body Boundary Conditions for the Euler Equations, ZAMM, 58 (1978), pp. 301-304.

6) Zhang, X.: Compressible Cavity Flow Oscillation Due to Shear Layer Instabilities and Pressure Feedback, AIAA J., 33 (1995), pp. 14041411.

7) Zhang, J. B.: Experimental and Computational Investigation of Supersonic Cavity Flows, Ph. D. Thesis, Dept. of Aeronautics and Astronautics, University of Tokyo, Japan, Sept. 2000, pp. 43-46.

8) Huang, P. G., Bradshaw, P. and Coakley, T. J.: Skin Friction and Velocity Profile Family for Compressible Turbulent Boundary Layers, AIAA J., 31 (1993), pp. 1600-1604.

9) Orkwis, P. D., Tam, C. J. and Disimile, P. J.: Observations on Using Experimental Data as Boundary Conditions for Computations, AIAA J., 33 (1995), pp. 176-178.

10) Krist, S. L., Biedron, R. T. and Rumsey, C. L.: CFL3D User's Manual (Version 5.0), NASA/TM-1998-208444, 1998, pp. 259-260.

11) Spalding, D. B. and Chi, S. W.: The Drag of a Compressible Turbulent Boundary Layer on a Smooth Flat Plate with and without Heat Transfer, J. Fluid Mech., 18, Pt. 1 (1964), pp. 117-143.

12) Coles, D.: The Turbulent Boundary Layer in a Compressible Fluid, Phys. Fluids, 7, 9 (1964), pp. 1403-1423.

13) Van Driest, E. R.: Turbulent Boundary Layer in Compressible Fluids, Aeronaut. J., 18, 3 (1951), pp. 145-160.

14) Van Driest, E. R.: The Problem of Aerodynamic Heating, Aeronaut. Eng. Rev., 15, 10 (1956), pp. 26-41.

15) Sommer, S. C. and Short, B. J.: Free-Flight Measurements of Turbulent-Boundary Layer Skin Friction in the Presence of Severe Aerodynamic Heating at Mach Numbers from 2.8 to 7.0, NACA TN 3391, 1955.

16) Baronti, P. O. and Libby, P. A.: Velocity Profiles in Turbulent Compressible Boundary Layers, AIAA J., 4 (1966), pp. 193-202.

17) Hopkins, E. J. and Inouye, M.: An Evaluation of Theories for Predicting Turbulent Skin Friction and Heat Transfer on Flat Plates at Supersonic and Hypersonic Mach Number, AIAA J., 9 (1971), pp. 993-1003.

18) Hopkins, E. J., Keener, E. R., Polek, T. E. and Dwyer, H. A.: Hypersonic Turbulent Skin-Friction and Boundary-Layer Profiles on Nonadiabatic Flat Plates, AIAA J., 10 (1972), pp. 40-48.

19) Fernholz, H. H. and Finley, P. J.: A Critical Commentary on Mean Flow Data for Two-Dimensional Compressible Turbulent Boundary Layers, AGARD-AG-253, 1980.

20) Cebeci, T. and Smith, A. M. O.: Analysis of Turbulent Boundary Layers, Academic Press, New York, 1974, p. 221.

21) Musker, A. J.: Explicit Expression for the Smooth Wall Velocity Distribution in a Turbulent Boundary Layer, AIAA J., 17 (1979), pp. 655657. 
22) Cornish, J. J., III: A Universal Description of Turbulent Boundary Layer Profiles with or without Transpiration, Research Rept. 29, Mississippi State Univ., Aero Physics Dept., 1960.

23) Bull, M. K.: Velocity Profiles of Turbulent Boundary Layers, Aeronaut. J., 73 (1969), p. 143.

24) Favre, A.: Equations des gaz Turbulents Compressibles, J. de Mecanique, 4, 3 (1965).

25) Wilcox, D. C.: Turbulence Modeling for CFD, DCW Industries, Inc., La Canada, California, 1993.

26) Baldwin, B. S. and Lomax, H.: Thin Layer Approximation and Algebraic Model for Separated Turbulent Flows, AIAA Paper 78-257, 1978.
27) Spalart, P. R. and Allmaras, S. R.: A One-Equation Turbulence Model for Aerodynamic Flows, AIAA Paper 92-0439, 1992.

28) Jones, W. P. and Launder, B. E.: The Prediction of Laminarization with a Two-Equation Model of Turbulence, Int. J. Heat Mass Transfer, 15, 2 (1972), pp. 301-314.

29) Wilcox, D. C.: Reassessment of the Scale-Determining Equation for Advanced Turbulence Models, AIAA J., 26 (1988), pp. 1299-1310.

30) Menter, F. R.: Two-Equation Eddy-Viscosity Turbulence Models for Engineering Applications, AIAA J., 32 (1994), pp. 1598-1605.

31) Townsend, A. A.: The Structure of Turbulent Shear Flow, Second Edition, Cambridge University Press, Cambridge, 1976. 